\title{
Research of Virtual Test Platform for Marine Instrument
}

\author{
Zheng Yi,Wang Zhongqiu \\ Shandong Provincial Key Laboratory of Ocean \\ Environment Monitoring Technology \\ Shandong Academy of Sciences Institute of \\ Oceanographic Instrumentation \\ Qingdao, China, 266001
}

\author{
Wang Juncheng ,Wang Hongru \\ Shandong Provincial Key Laboratory of Ocean \\ Environment Monitoring Technology \\ Shandong Academy of Sciences Institute of \\ Oceanographic Instrumentation \\ Qingdao, China, 266001
}

\begin{abstract}
Virtual test Platform for marine instrument could provide visualized and interactive simulation solution for the development process. It could be used for virtual test or virtual experiment of mechanical or electro characters of marine instrument. The marine virtual instrument test environment is established combining with data management technology, visualization technologies and corresponding software and hardware system integration technology. Further, the modeling methods of marine instrument virtual model are proposed, including the geometric model, physical model and behavior model. The marine instrument virtual experiment platform prototype is established based on the research of key technologies, and the prototype is verified by using the example of submerged buoy development process of marine hydrodynamic environment monitoring.)
\end{abstract}

Keywords-Marine Instrument, Virtual Experiment, Virtual Test, Modeling

\section{INTRODUCTION}

With the continual improvement of computer software and hardware performance, virtual reality technology and equipment becomes practical [1], and the test technology based on virtual reality display shows more and more attractiveness. But this kind of research work has only just started, some applications and other aspects of the design is yet to be carried out, such as theoretical foundation, support technology and design application.

Right now, this virtual test platform is commonly studied and applied in automobile, aerospace and weapon development area. In the automobile company MTS, Vehicle Virtual Test System (VVTS) has been used to reproduce the actual external environment, so that the comprehensive performance could be analyzed and evaluated in laboratory to find out the design flaws, as a result, the automobile development cycle could be shortened. NASA developed a virtual engineering environment as Virtual Test Bed (VTB) to research the function of space shuttles appeared now and in next generation [2, 3]. This virtual test bed adopts the High-Level Architecture (HLA) as the main environment, and could simulate the launching process of space shuttle.

A virtual test bed is developed by U.S. navy to design advanced power system [4]. This virtual test bed could help the group members to participate in the interdisciplinary design and analysis of virtual prototyping simultaneously.
Figure 1. is the application of this VTB system used in some type of combat vehicles and airplane power system.
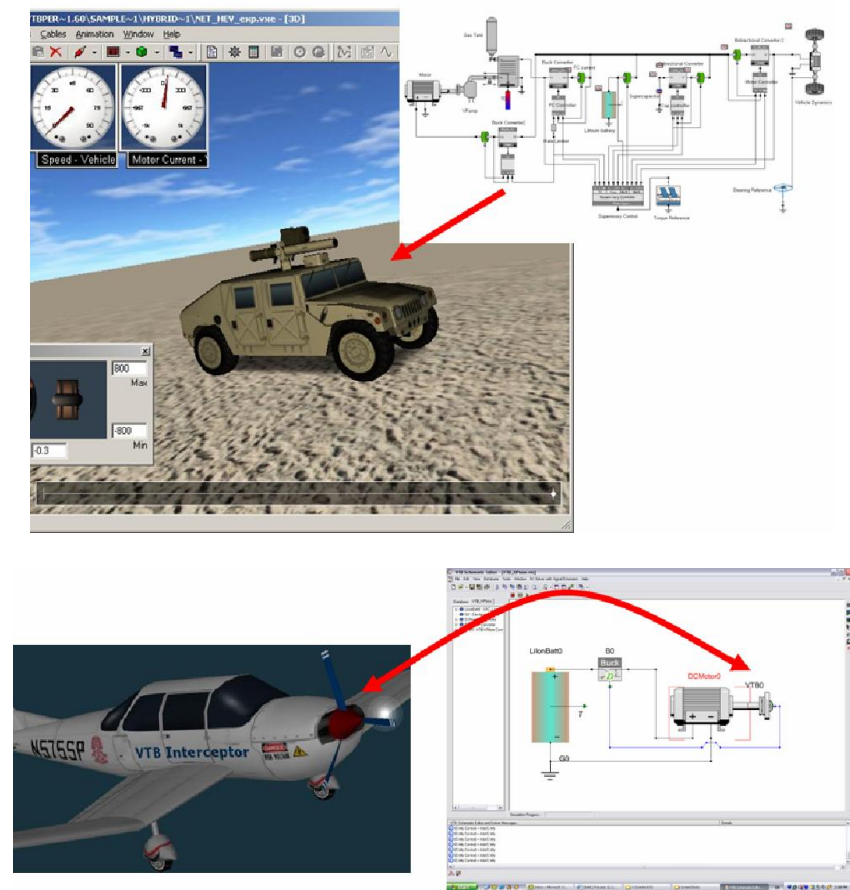

Figure 1. Dynamic simulation of fuel cells

It can be seen from the literatures above that the virtual test techniques have been studied and applied in the fields of automotive and aerospace area, but less in marine equipment. There are more complexities in marine environment need to be considered to model the virtual test bed, so the experiences in other fields could not be transferred easily, but the common techniques could be learned from each other.

\section{REAERCH CONTENTS}

\section{A. System structure of marine institute virtual test platform}

The complexity of the marine environment and objective law of development of scientific research determines that the virtual test platform structure must take into account current research and future extensions, such as adding salt water corrosion process in the future evolution of the model simulation analysis. Learning from the U.S. Department of 
Defense's HLA, modular and expandable test platform architecture system is considered, as shown in Figure 2.

In this system structure, marine instrument researchers could interact with each other through application I/O and visualization module in profession and logic layer. The profession and logic layer is supported by the support layer and the data management module in this layer, and could be expanded easily by the expand port.

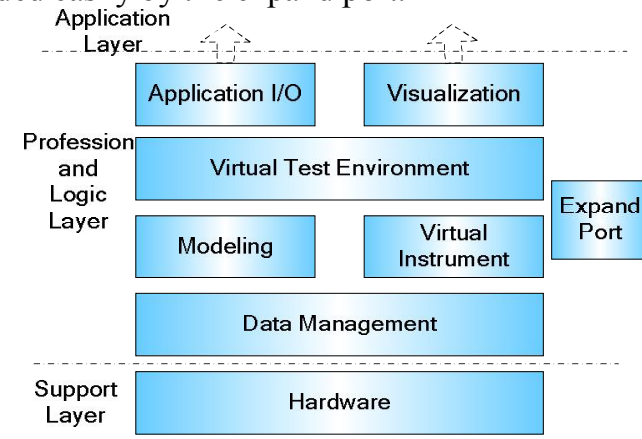

Figure 2. System structuure of marine instrument virutal test platform

\section{B. Details of key technologies of marine institute virtual test platform}

In order to realize the system structure, some details techniques must be taken into account, listing as below:

(1) The software and hardware construction and the system integration research.

(2) The modeling methods research of marine instrument virtual experiment objects and virtual test environment.

(3) The virtual test methods research of virtual experiment on marine instrument virtual test platform.

(4) Research of comprehensive expression method of visualization of marine instrument virtual models, test environment and test process.

(5) Data management of marine instrument virtual test platform.

Based on the techniques above, the marine institute virtual test platform prototype is established, including the object library module that can import 3D geometries as standard object, analyzing module that can apply boundaries and affecting factors to other connected analysis software and the virtual visualization module that can show the results in $3 \mathrm{D}$ views.

\section{4 EXAMPLES OFAPPLICATION}

In order to verify the marine institute virtual test platform prototype, the example of submerged buoy development process of marine hydrodynamic environment monitoring is considered. In this case, the submerged buoy will work in the sea anchored by cable, but as the effects of wave and flow of the sea water, the submerged buoy will vibrate, so the other facilities mounted on the buoy will not work functional, so before the actual buoy is manufactured, it can be tested in the virtual test platform to help adjust the design parameters.

The process is that, the object 3D geometry of submerged buoy is modeled by CAD software, and the model is imported into the platform object library as standard model, and then the object model and boundary conditions are exported to the analyze software in standard form to derive the deformation mode results. At last, the analyze results can be analyzed in virtual platform. The submerged buoy is shown in Figure3. The battery and suspension ring of the buoy are added one by one to inspect the vibration variation. The buoy model without any extra component is named M1, and the one with battery is named M2, and the one with battery and suspension ring is named M3. The vibration analysis results are shown in Figure 4 and Figure 5.
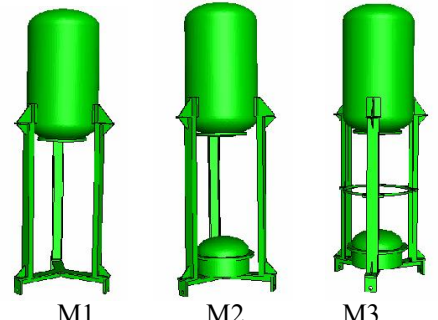

Figure 3. The CAD model of aluminum alloy submerged buoy
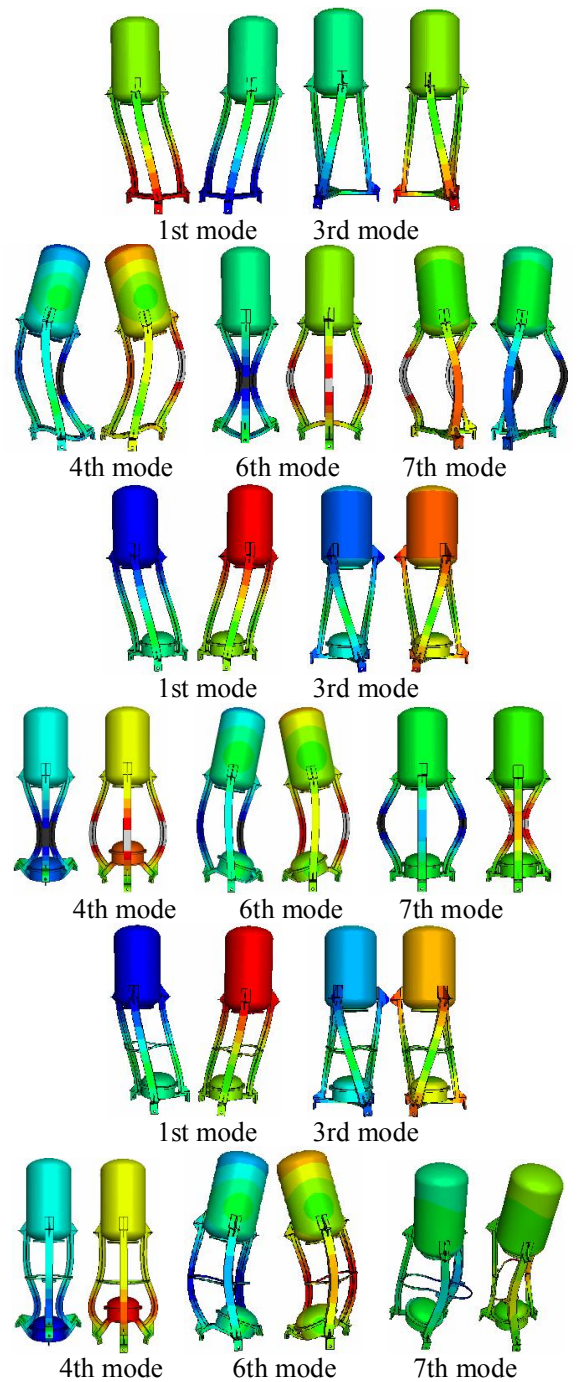

Figure 4. The first 7 th vibration changing of different mode structure 
Comparing M1 with M2, it can be seen from Figure 5 that, when the battery is added to buoy, the low frequency vibration modes don't change, but the higher frequency vibration mode are different, the 6th mode in M1 moves forward to the 4th mode in M2, and the 9th mode (not shown in Figure 5) in M1 moves forward to the 7th mode. Comparing M2 with M3, there is no obvious different, but the 7 th mode. Considering the frequency variation, it can be seen from Figure 6 that, when the battery is added, the frequency of the same vibration mode decreases since the system mass increases, as is shown in the comparison of M1 and M2; and when the suspension ring is added, the frequency of the same vibration mode increases a little, since the system rigid increases. The trend coincides with the

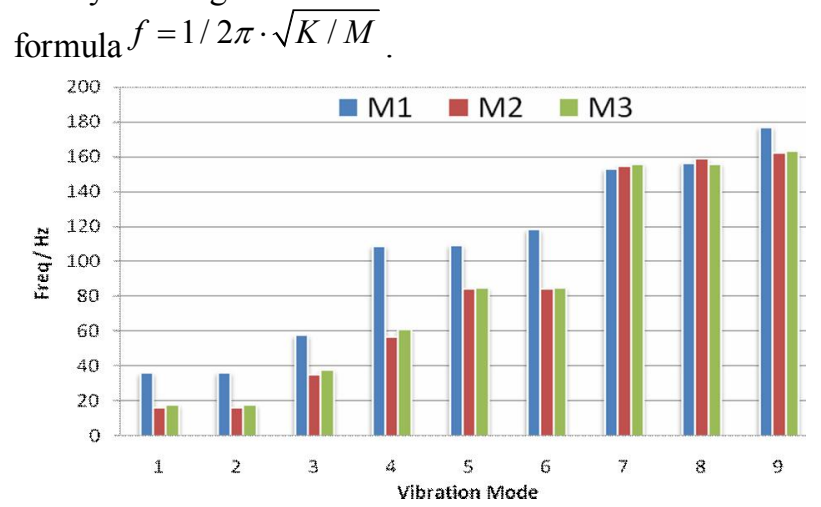

Figure 5. The first 9 mode's frequency of different mode structure

\section{SUMMARIES}

Marine instruments virtual test platform has the following advantages:

(1) Low cost of experiment. It is needed to be funded for one time and could be used for long-term. And it can be adjusted as needed;

(2) Regardless constraints of time and space. The scale of space and time can be changed, so that the details of the process can be studied in any test time;

(3) Capable of extrapolation for certain characteristic. Some of the actual test results are not able to be obtained, but can be simulated and predicted in the virtual test platform;

(4) Convenient to research the affects of interference and test parameters in the test process on the test results. The affect of different parameters and process interference on the properties of test object during the test could be observed, and a variety of intermediate results or unexpected results could also be observed.

(5) Easy to understand the behavior and mechanism during the test. By the replay, prediction and slow display of the test process results, the deep understanding of the test object and test process can be learned. And the virtual test platform could also be used for personnel training for new or complex test tasks or test object.

In the current stage of development, marine instrument virtual test platform could not replace the prototype test, but provide a realistic test environment and a variety of tools and instruments for the developer, and allow the developers to do experiments and test the marine instrument prototype as more as possible before it is completed and improve the design, so that the number of iterations and prototype can be reduced in research and development process. As a result, the success rate of the first to achieve can be increased, and development cycles can be shortened, the development costs could be reduced, and the reliability and functionality can be improved.

\section{ACKNOWLEDGMENT}

This paper was supported by the National Natural Science Foundation of China under Grant 40806044 and Supported by the Qingdao Science and Technology Planning Project Fund, China under Grant 10-3-4-9-2-jch.

\section{REFERENCES}

[1] Jayaram S, Connacher H, Lyons K. Virtual Assembly using virtualreality techniques. Computer Aided Design [J], 1997, 8(29), PP: 575584

[2] L. Rabelo, J. Sepulveda, and J. Park. Range Safety Modeling in the Virtual Test Bed

[3] José Sepúlveda, Frank Riddick, Cary Peaden. Implementing the high level architecture in the virtual test bed [A]. Proceedings of the 2004 Winter Simulation Conference [C], 2004, PP: 1444-1451

[4] The Board of Trustees of the University of South Carolina. Virtual Test Bed [EB/OL]. http://vtb.engr.sc.edu/ 2007-3-20

[5] Denis Gracanin, Kimon P. Valavanis , Maja Matijas evic. Virtual environment test bed for autonomous underwater vehicles. Control Engineering Practice [J], 1998. 6(5), PP: 653-660.

[6] Gracanin D, Matijasevic, M, Valavanis, K.P. Virtual environment test bed for underwater robotics applications [A]. Proceedings of 8th International Conference on Advanced Robotics [C]. 1997, PP: 793797. 